\title{
CBCT-Assessment of Morphological Characteristics of the Alveolar Bone in the Aesthetic Zone
}

\author{
Irena Georgieva ${ }^{1}$, Stefan Peev ${ }^{2}$ \\ Department of Periodontology and Dental Implantology, Medical University of Varna, Bulgaria
}

\begin{abstract}
The total number of the assessed teeth on Cone-beam computed tomography(CBCT) $x$-ray images of the test patients in our investigation is 528 frontal teeth (176 central incisors, 176 lateral incisors and 176 canines). In every one of the assessed teeth are made two measurements in $\mathrm{mm}$ - measurement of the height of the facial and the height of the palatal alveolar bone plate. The reference points for these two measurements are the most apical point of the apex of the root and the most coronary point of the marginal alveolar edge on the vestibular and palatal side. The analysis of the results showed that there is no significant difference between the mean hight of labial and palatal bone.
\end{abstract}

Keywords: aesthetic zone, maxilla, alveolar bone plate, CBCT, implant therapy, aesthetic risk

\section{Introduction}

Nowadays, in the contemporary dental medicine, the aesthetic requirements of the pacient and dentist for treatment plan and dental treatment, increase more and more. The characteristics of the gingival and bone architectonics have a decisive meaning for the aesthetic risk of the dental treatment, as well as for the conservative and surgical treatment of the periodontal diseases and for the dental implant therapy.

Dental implants are used often in the contemporary dental treatment for the restoration of the loss of frontal teeth in the aesthetic zone of the maxilla. Implant therapy in the anterior maxilla is considered an advanced or complex procedure and requires comprehensive preoperative planning and precise surgical execution. The continuous researches in this area suggest different treatment options to the clinicians, that facilitate implant therapy in the cases of alveolar bone deficiency in the aesthetic zone of the maxilla. The height and the thickness of the alveolar bone plate are decisive in the preoperative planning.

The primary stability of the implant depends a lot on the quantity of the present alveolar bone. That is why it is very important to be considerd the width of the alveolar ridge and the height of the bucal and palatal alveolar bone plate before planninig implat therapy in the aesthetic zone of the maxilla.

The implant therapy is possible only after precise clinical and radiographic assessment of the area, that would gonna be restored. The exact assessment of the height, width and angulation of the bone is important for the correct treatment planning, which hand to correct determination of the prognosis and reduce the aesthetic risk.

\section{Literature Survey}

The most used -x-ray methods for determination of the alveolar bone level in dental practice are the intraoral periapical radiography (IOPR) and the panoramic radiography
(OPG). Eickholz and Hausmann showed that radiographic assessment using peri-apical radiographs tends to underestimate the amount of bone loss by $1.41 \pm 2.58$ mm.Furthermore in the IOPR and OPG, there is overlap of the images, especially of the area of the facial and palatal alveolar bone plate, which does not allow to be assessed the height of the vestibular and palatal alveolar bone. It is not possible to be assessed the width of the alveolar ridge with these radiographic methods. $\left({ }^{2}\right)$

Cone-beam computed tomography (CBCT) is a technology that is necessary for the correct 3-dimensional diagnostic of the anatomic characteristics of the alveolar bone in the aesthetic zone of the maxilla, because it presents in details the anatomic features and the resorptive changes in the alveolar bone in comparison with the IOPR and OPG. $\left({ }^{1}\right)$

In comparison with the conventional radiographic methods, the use of CBCT technology has proved priority in the diagnostic of the periodontal diseases and in the preoperative planning of implant therapy, because it gives an advantage, that the examined area can be assessed and analysed 3 -dimensional. $\left({ }^{3}\right)$

The x-ray images, received by the CBCT have bigger resolution in comparison with these, received by the conventional radiographic methods. The CBCT gives 3dimensional $x$-ray image of the assessed area and in relation with the alveolar bone it is more detailed in comparison with the 2-dimensional panoramic radiography and IOPR, that may lead to difficulty in the assessment of the level of the facial and the palatal alveolar bone, because of the overlapping of the images of anatomic characteristics of the bone. That may lead to errors in identifying reliable anatomical reference point in the diagnostics. It is not possible to be determined the height and the thickness of the vestibular and palatal alveolar bone with the panoramic radiography. $\left({ }^{4}\right)$

CBCT has $80-100 \%$ sensitivity in examination and determination of the alveolar bone loss, until the 


\section{International Journal of Science and Research (IJSR) \\ ISSN (Online): 2319-7064}

Index Copernicus Value (2013): 6.14 | Impact Factor (2014): 5.611

conventional radiographic methods show about 63-67\% sensitivity. $\left({ }^{5}\right)$

CBCT is variant of the traditional cone tomography (CT), but has some advantages such as the reduce radiation exposure and low cost in comparison with the conventional CT. $\left({ }^{6}\right)$

In implant therapy due to the CBCT can be taken a decision for most proper place for the implant, the proper size of the implant and the process of osseointegration can be followed in time. Not only due to CBCT can be escaped a lot of complications during the surgical manipulation, but also in some situations can be minimized the necessity of additional procedures like bone or soft tissue augmentation. $\left(^{7}\right)$

\section{Material and Methods}

The aim of this epidemiological study is to be determine the height of vestibular and palatal alveolar bone plate of the maxillary frontal teeth in the aesthetic zone of the upper jaw. Also to be generalized the variations in the height of the facial and palatal alveolar bone wall in these teeth, to be compared and generalized the differences between the measurements in the case of right and left maxillary frontal teeth, and the differences in measurements between males and females. To determine the significance of these variations for the aesthetic risk for implant therapy in this zone.

The study includes only upper frontal teeth in the aesthetic zone of the maxilla of 88 individuals, assessed over the base of their radiographic images, received by $\mathrm{CBCT}$.

In the test group are included patients who respond to the following criteria:

- all participants in the study have permanent dentition in the frontal sextant of the maxilla

- all of them have intact upper frontal teeth - there is no missing or decayed teeth in the upper frontal sextant

- there is no prosthetically restored upper frontal teeth with crowns or bridges

- in the radiographic image of all of the participants in the test group, there are no resorptive pathological changes in the zone of the interdental alveolar bone

From the test group are excluded:

- CBCT images of patients who have missing frontal upper teeth

- CBCT images of patients with present primary frontal upper teeth

- CBCT images of patients whose frontal upper teeth in the zone of the maxilla are restored with crowns or bridges

- CBCT images of patient who have brackets and as well

- CBCT images of patients with periodontal diseases and respectively pathological resorption of the alveolar bone in the aesthetic zone of the maxilla

In the test group there are totally 88 patients ( 36 males and 52 females) on the age between 17 and 68 years old (average age 39 years). The sex distribution shows 59, 1\% females and 40, 9 males.

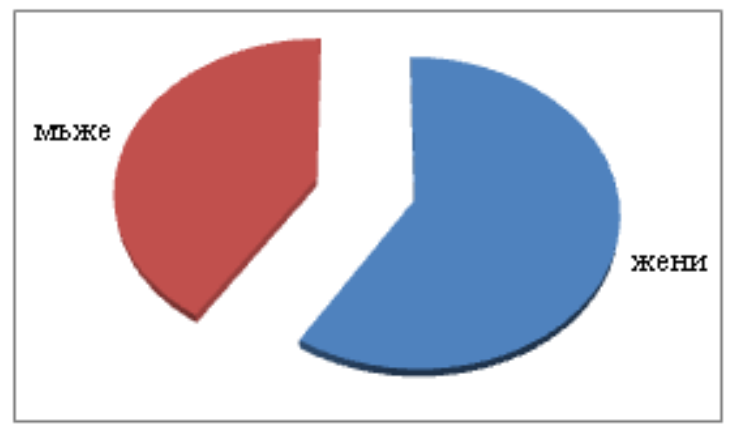

Figure 1: Distribution of the test group to males and females

The total number of the teeth, which are included in the study and which alveolar bone is analyzed on the CBCT radiographic images, is 528 maxillary frontal teeth (176 central incisors, 176 lateral incisors and 176 canines).

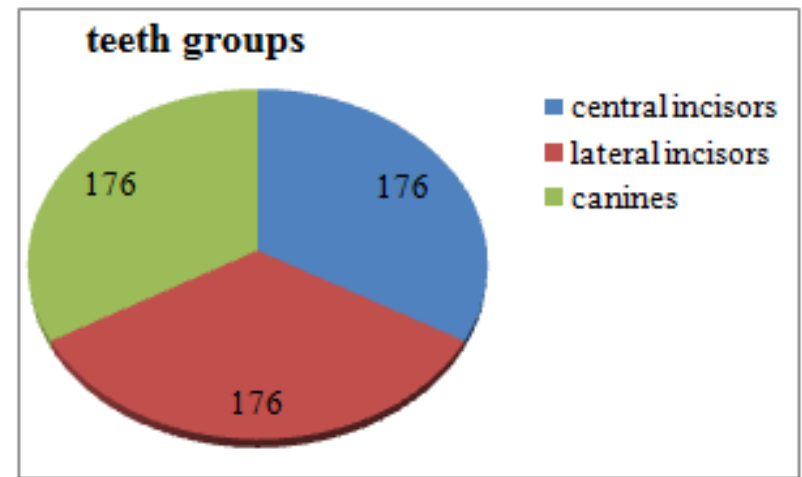

Figure 2: Distribution on groups of the assessed frontal maxillary teeth

The CBCT radiographic images that are used, are from data base of Medical University of Varna and are made in period between January 2015 and June 2015. The name, age and sex of each patient are registered in the data base. With the help of the software of the CBCT are assessed and analyzed the height of the vestibular and height of palatal alveolar cortical bone plate of upper frontal teeth. The CBCT that we used for this epidemiological study is Planmeca, Promax 3D Max, Findland c $96 \mathrm{kV}$ and $12 \mathrm{~mA}$.

There are made two measurements to all of the assessed upper frontal teeth. On CBCT images to all of the assessed teeth first we measure the height of the vestibular alveolar bone plate and after that the height of the palatal one. The measurement is done through the center of the sagittal plane of each of the frontal upper teeth. As referent points are used the apex of the tooth root and the most coronary point of the alveolar marginal edge, respectively of the vestibular and palatal one alveolar bone plate. The distance from the most apical point of the root and the most coronary point of the vestibular and palatal marginal alveolar edge is measured in $\mathrm{mm}$ to all of the assessed maxillary frontal teeth. 


\section{International Journal of Science and Research (IJSR) \\ ISSN (Online): 2319-7064}

Index Copernicus Value (2013): 6.14 | Impact Factor (2014): 5.611

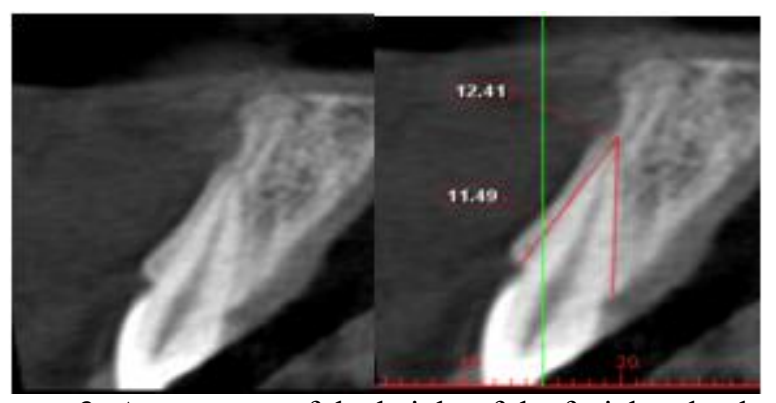

Figure 3: Assessment of the height of the facial and palatal alveolar bone plate of central incisor

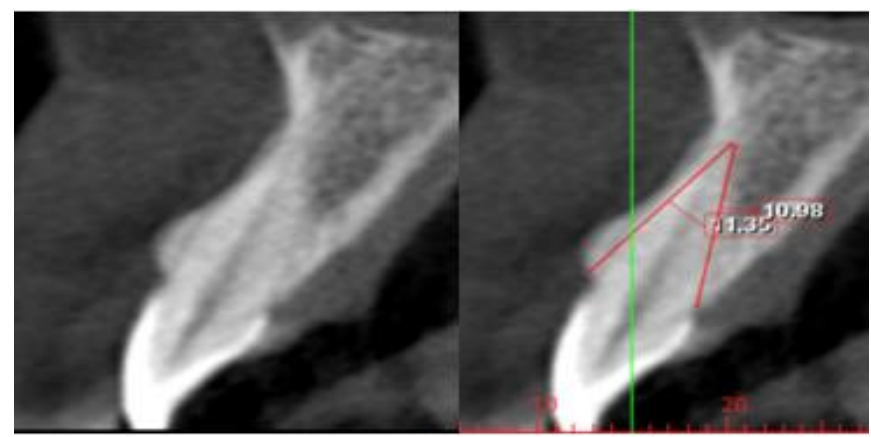

Figure 4: Assessment of the height of the facial and palatal alveolar bone plate of lateral incisor

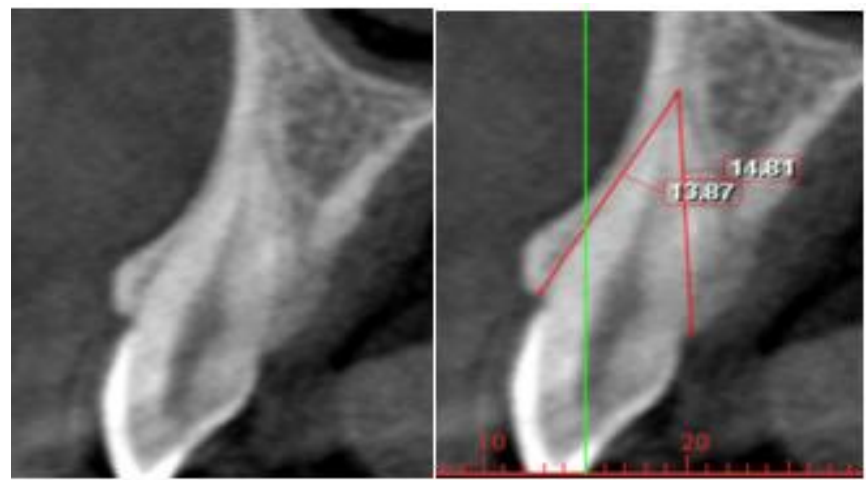

Figure 5: Assessment of the height of the facial and palatal alveolar bone plate of canine

The names, the age and the sex of the participants in the study and the results from measurements for each of the assessed frontal teeth from tooth 13 to tooth 23 are saved in a table. For the analysis of CBCT images is used $3 \mathrm{~d}$ software Romexis (Planmeca, Promax 3D, Findland).

\section{Results}

The results from the study indicative that in the central incisors, the mean height of the vestibular alveolar bone plate is $10,64 \mathrm{~mm}$ and vary between $6,02-14,56 \mathrm{~mm}$, and the mean height of the palatal one is 11,68 and vary between 6,36-15,59 mm.

For comparison the results for the lateral incisors show, the mean height of the vestibular alveolar bone plate is 11,11 $\mathrm{mm}$ and vary between $6,79-16,01 \mathrm{~mm}$, and the mean height of the palatal one is $11,23 \mathrm{~mm}$ and vary between $6,60-16,21 \mathrm{~mm}$.

For the canines the results indicate, that the mean height of the vestibular alveolar bone plate is $13,87 \mathrm{~mm}$ and vary between 7,64 - 21,40 $\mathrm{mm}$, and the mean height of the palatal one is $14,39 \mathrm{~mm}$ and vary between $6,85-21,44 \mathrm{~mm}$.

In the aesthetic zone of the maxilla, the height of the facial and palatal alveolar bone plate is biggest in the maxillary canines. In one study Goodacre determine that when plan implant therapy in the aesthetic zone of the maxilla, the height of the alveolar bone must be $\geq 10 \mathrm{~mm}$, because the different implants with length $\geq 10 \mathrm{~mm}$ show long-term stability in comparison with the shorter implants. $\left({ }^{8}\right)$

In this connection in our measurements and when we determine the distribution of the height of the vestibular alveolar bone plate, we used the limit value $10 \mathrm{~mm}$ and that is why our results are generalized in two groups: respectively with height of the alveolar bone under $10 \mathrm{~mm}$ and results with height of alveolar bone over $10 \mathrm{~mm}$.

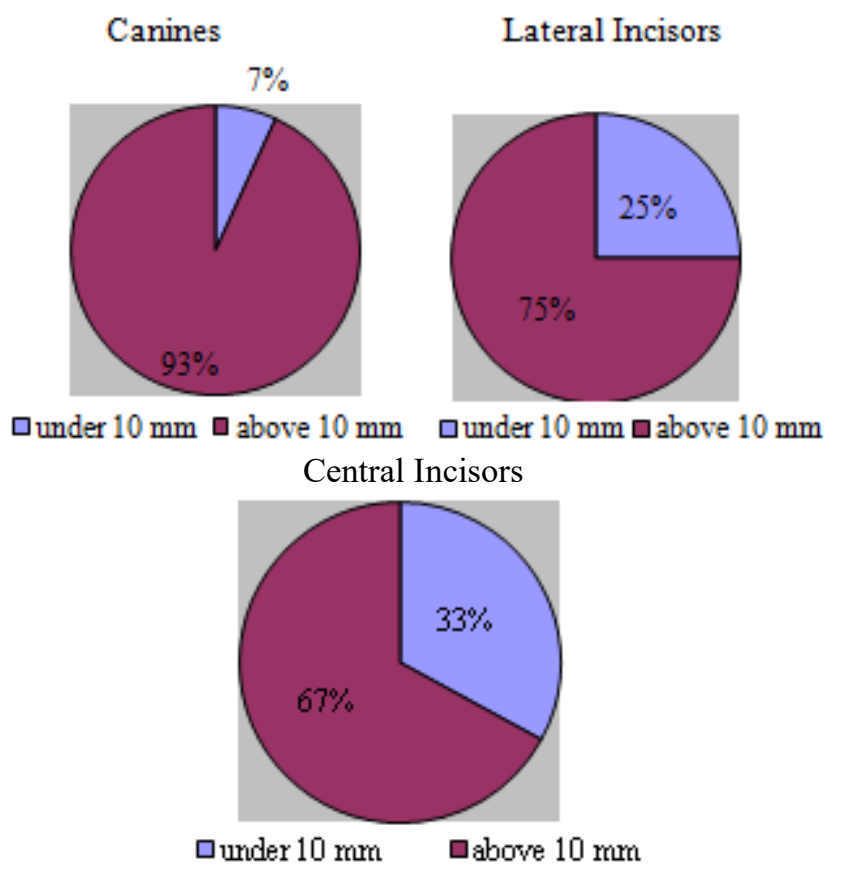

Figure 6: Distribution of the height of the facial alveolar bone above and under $10 \mathrm{~mm}$ in the different groups of maxillary frontal teeth

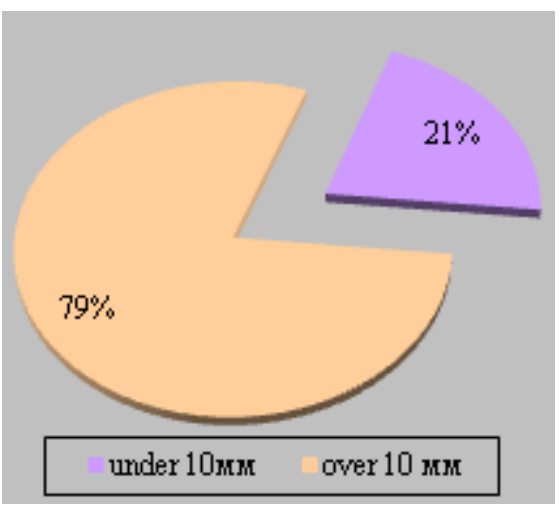

Figure 7: Generalized results of the distribution of the height of the facial alveolar above and under $10 \mathrm{~mm}$ in all groups of the maxillary frontal teeth

The height of the facial alveolar bone plate become higher in direction from the central incisors to lateral incisors and canines. 


\section{International Journal of Science and Research (IJSR) \\ ISSN (Online): 2319-7064}

Index Copernicus Value (2013): 6.14 | Impact Factor (2014): 5.611

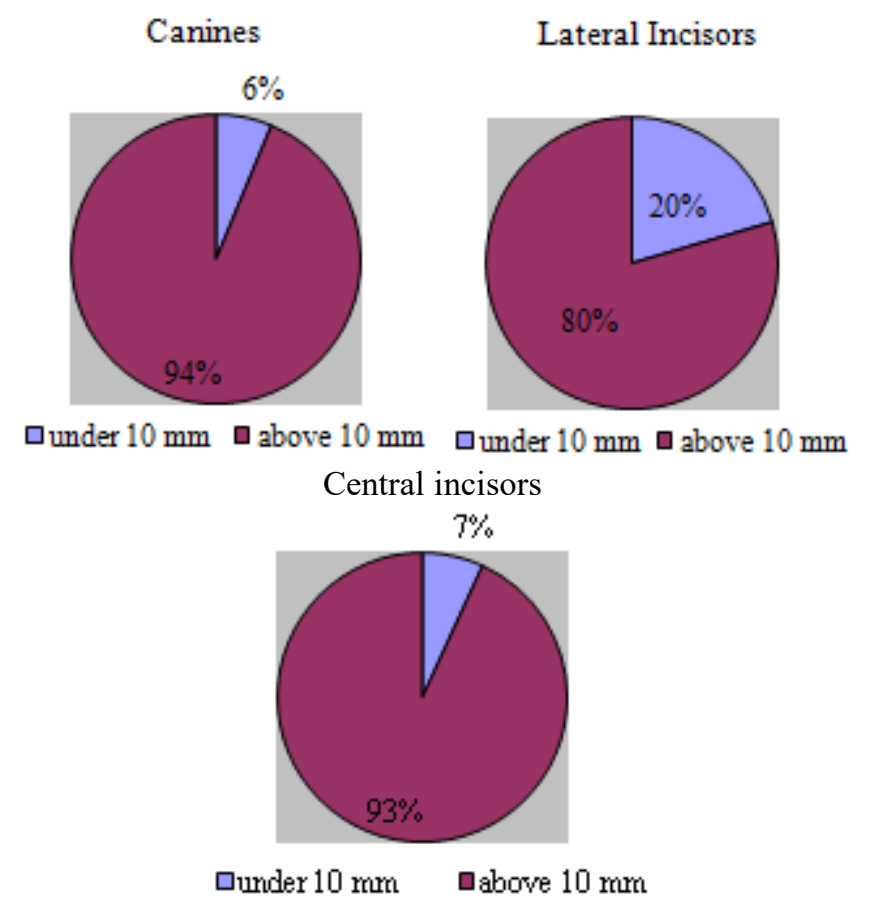

Figure 8: Distribution of the height of the palatal alveolar bone plate under and above $10 \mathrm{~mm}$ in the different groups of maxillary frontal teeth

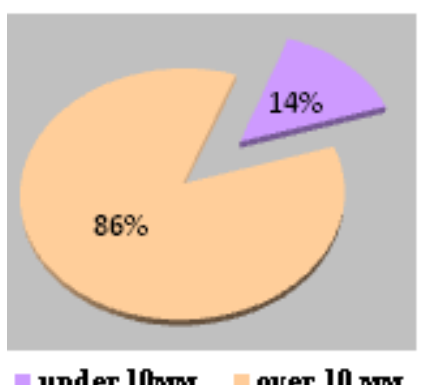

Figure 9: Generalized results of the distribution of the height of the palatal alveolar above and under $10 \mathrm{~mm}$ in all groups of the maxillary frontal teeth

The height of the palatal alveolar bone plate become higher in direction from the lateral incisors through the central incisors and it is highest in the maxillary canines. The data of the hold study with the measurements of the height of facial and palatal alveolar bone plate in the maxillary frontal teeth are generalized in the following table 1 :

Table 1: Mean values of the height of the facial and palalatal alveolar bone in the maxillary frontal teeth

\begin{tabular}{|c|c|c|c|c|c|c|}
\hline \multirow{2}{*}{} & \multicolumn{2}{|c|}{$\begin{array}{c}\text { Central Incisors } \\
(\mathrm{n}=176)\end{array}$} & \multicolumn{2}{c|}{$\begin{array}{c}\text { Lateral Incisors } \\
(\mathrm{n}=176)\end{array}$} & \multicolumn{2}{c|}{$\begin{array}{c}\text { Canines } \\
(\mathrm{n}=176)\end{array}$} \\
\cline { 2 - 8 } & facial & palatal & facial & palatal & facial & palatal \\
\hline Mean value & 10,64 & 11,68 & 11,11 & 11,23 & 13,87 & 14,39 \\
\hline minimum & 6,02 & 6,36 & 6,79 & 6,6 & 7,64 & 6,85 \\
\hline maximum & 14,99 & 15,59 & 16,01 & 16,21 & 21,4 & 22,89 \\
\hline
\end{tabular}

Table 2: Comparison of the mean values of the height of the facial and palatal alveolar bone plate in the frontal maxillary teeth in the left and right half of the dentition

\begin{tabular}{|c|c|c|c|c|c|c|}
\hline \multirow{2}{*}{} & \multicolumn{2}{|c|}{$\begin{array}{c}\text { central incisors } \\
(\mathrm{n}=176)\end{array}$} & \multicolumn{2}{c|}{$\begin{array}{c}\text { lateral incisors } \\
(\mathrm{n}=176)\end{array}$} & \multicolumn{2}{c|}{$\begin{array}{c}\text { Canines } \\
(\mathrm{n}=176)\end{array}$} \\
\cline { 2 - 7 } & right & left & right & left & right & left \\
\hline facial & 10,67 & 10,61 & 11,19 & 11,03 & 14,04 & 13,69 \\
\hline palatal & 11,66 & 11,7 & 11,29 & 11,17 & 14,41 & 14,38 \\
\hline
\end{tabular}

The data in table 2 are indicative that there are no statistic differences between the height of the facial and palatal alveolar bone plate of the maxillary frontal teeth from one group, respectively in the left and right half of the dentition of the upper jaw.

Table 3: Comparison of the mean values of the height of the facial and palatal alveolar bone plate in the frontal maxillary teeth in males and females

\begin{tabular}{|l|c|c|c|c|c|c|}
\hline & \multicolumn{2}{|c|}{$\begin{array}{c}\text { central incisors } \\
(\mathrm{n}=176)\end{array}$} & \multicolumn{2}{c|}{$\begin{array}{c}\text { lateral incisors } \\
(\mathrm{n}=176)\end{array}$} & \multicolumn{2}{c|}{$\begin{array}{c}\text { Canines } \\
(\mathrm{n}=176)\end{array}$} \\
\hline & females & males & females & males & females & males \\
\hline facial & 10,44 & 10,93 & 10,87 & 11,61 & 12,93 & 15,22 \\
\hline palatal & 11,26 & 12,29 & 10,85 & 11,95 & 13,35 & 15,90 \\
\hline
\end{tabular}

The data in table 3 are indicative, that the height of the facial and palatal alveolar bone plate is higher in all maxillary frontal teeth of males and the differences are most considerable in the zone of the canines. In males and females the mean height of the facial alveolar bone wall become higher in direction from the central incisors to canines. In comparison, in males and females, the mean value of the height of the palatal alveolar bone plate become higher in direction from the lateral incisors through the central incisors and it is highest in the maxillary canines.

\section{Conclusion}

There is no significant difference between the mean hight of labial and palatal bone.

\section{References}

[1] Variations in the vestibular cortical bone of permanent canine teeth in orthodontic patients. A comparative study: linear tomography vs. CBCT - María E. Mateu, M.E. Martínez, H. Dagum, Sandra C. Benítez Rogé, Gabriela I. Bruno, Pedro Hecht, Alejandra A. Folco Acta Odontol. Latinoam. 2014 Vol. 27 N 2 / 2014 / 5862

[2] CBCT - A Positive Amelioration In Periodontics - Dr. Monali A. Shah, Dr. Sneha S. Shah, Dr. Deepak H. Dave

[3] Evaluation Of FacialL Alveolar Bone Dimension Of Maxillary Anterios And Premolar Teeth - Int J Oral Maxillofac Implants. 2012 Nov-Dec;27(6):1514-9

[4] Diagnostic Applications of Cone-Beam CT forPeriodontal Diseases - Yousef A. AlJehani International Journal of Dentistry, Volume 2014, Article ID 865079,5 pages

[5] Detection of periodontal bone loss using cone beam CT and intraoral radiography - $\mathrm{K}$ de Faria Vasconcelos, KM Evangelista, CD Rodrigues, C Estrela, TO de Sousa, and MAG Silva -Dentomaxillofac Radiol. 2012 Jan; 41(1): 64-69 


\section{International Journal of Science and Research (IJSR) \\ ISSN (Online): 2319-7064}

Index Copernicus Value (2013): 6.14 | Impact Factor (2014): 5.611

[6] Three-dimensional imaging in periodontal diagnosis Utilization of cone beam computed tomography Ranjana Mohan, Archana Singh, Mohan Gundappa Journal of Indian Society of Periodontology, Vol. 15, No. 1, January-March, 2011, p. 11-17

[7] Alveolar bone morphology under the perspective of the computed tomography: Defining the biological limits of tooth movement - Daniela Gamba Garib, Marulia Sayako Yatabe, Terumi Okada Ozawa, Omar Gabriel da Silva Filho - Dental Press J Orthod., 2010 Sept-Oct;15(5):192205

[8] Clinical complications with implants and implant prostheses. - Goodacre CJ, Bernal G, Rungcharassaeng K, Kan JY - J Prosthet Dent. 2003 Aug;90(2):121-32

\section{Author Profile}

Irena Georgieva, DMD - Assistant Professor at Department of Periodontology and Dental Implantology, Medical University of Varna, Bulgaria

Stefan Peev DMD, PhD, DSc - Professor (Associate), Head of Department of Periodontology and Dental Implantology, Medical University of Varna, Bulgaria, Director of University Dental Clinic

- Varna 